\title{
Signature of Saturn's auroral cusp: Simultaneous Hubble Space Telescope FUV observations and upstream solar wind monitoring
}

\author{
Jean-Claude Gérard, ${ }^{1}$ Emma J. Bunce, ${ }^{2}$ Denis Grodent, ${ }^{1}$ Stanley W. H. Cowley, ${ }^{2}$ \\ John T. Clarke, ${ }^{3}$ and Sarah V. Badman ${ }^{2}$ \\ Received 18 February 2005; revised 7 July 2005; accepted 29 July 2005; published 11 November 2005.
}

[1] Model simulations by Bunce et al. (2005a) have shown that direct precipitation of electrons in Saturn's dayside cusp regions is not capable of producing significant FUV aurora. Instead, they suggested the possibility that the FUV bright emissions sometimes observed near noon are associated with reconnection occurring at the dayside magnetopause, possibly pulsed, analogous to flux transfer events seen at the Earth. Pulsed reconnection at the low-latitude dayside magnetopause when the IMF is directed northward (antiparallel to Saturn's magnetic field lines) is expected to give rise to pulsed twin-vortical flows in the magnetosphere and hence to bipolar field-aligned currents centered in the vortical flows closing in ionospheric Pedersen current. In the case of southward IMF and high-latitude lobe reconnection the model predicts that the vortical flows are displaced poleward of the open-closed field line boundary with reversed fieldaligned currents compared with the former case. During January 2004, a unique campaign took place during which magnetic field and plasma instruments on board the CassiniHuygens spacecraft measured the in situ solar wind and embedded interplanetary magnetic field while the Hubble Space Telescope simultaneously observed the far ultraviolet aurora in Saturn's southern hemisphere. The IMF was highly structured during this interval. The electric potential at Cassini is estimated from solar wind magnetic field and velocity measurements for the case of low-latitude or lobe reconnection. We show that a dayside FUV signature of intense electron precipitation is found poleward of or along the main oval during a period of minor compression period when the dayside reconnection voltage is estimated to be $\sim 30-100 \mathrm{kV}$. Overall, we find that the conceptual model of Bunce et al. (2005a) provides a good estimate of the UV brightness and power for the case of northward IMF but somewhat underestimates the power for the southward IMF case, except if the speed of the vortical flow is larger than its value in the nominal model.

Citation: Gérard, J.-C., E. J. Bunce, D. Grodent, S. W. H. Cowley, J. T. Clarke, and S. V. Badman (2005), Signature of Saturn's auroral cusp: Simultaneous Hubble Space Telescope FUV observations and upstream solar wind monitoring, J. Geophys. Res., 110, A11201, doi:10.1029/2005JA011094.

\section{Introduction}

[2] The Earth's dayside cusp is the magnetospheric region where magnetosheath plasma has direct access to the ionosphere. The magnetospheric cusp therefore plays an important role as the region of most direct connection between the ionosphere and the interplanetary medium through reconnection. It exists whether the interplanetary magnetic field is northward or southward. Reconnection between magnetospheric and interplanetary magnetic field

\footnotetext{
${ }^{1}$ Laboratoire de Physique Atmosphérique et Planétaire, Université de Liège, Liège, Belgium.

${ }^{2}$ Department of Physics and Astronomy, University of Leicester, Leicester, UK. USA.

${ }^{3}$ Center for Space Physics, Boston University, Boston, Massachusetts,

Copyright 2005 by the American Geophysical Union. 0148-0227/05/2005JA011094
}

(IMF) lines is believed to occur whenever their directions (or at least one component) are antiparallel [Onsager and Fuselier, 1994; Fuselier et al., 1997]. During southward IMF conditions, magnetic field lines in the subsolar region can connect to the solar wind magnetic field and become open. Reconnection can take place at the high-latitude magnetopause during northward IMF conditions. During intermediate conditions with a small northward and dominating east-west $\left(B_{y}\right)$ component of the IMF, a mixed situation with reconnection at the high- and the low-latitude region may occur simultaneously [Reiff and Burch, 1985]. In situ measurements of precipitated particles have confirmed that the location and spectrum of particle precipitation in the cusp depend on the IMF orientation [Newell et al., 1989] and that they are indicators of reconnection at the magnetopause [Onsager et al., 1993].

[3] Milan et al. [2000] observed ultraviolet emission near local noon, poleward of the dayside auroral oval, with the 


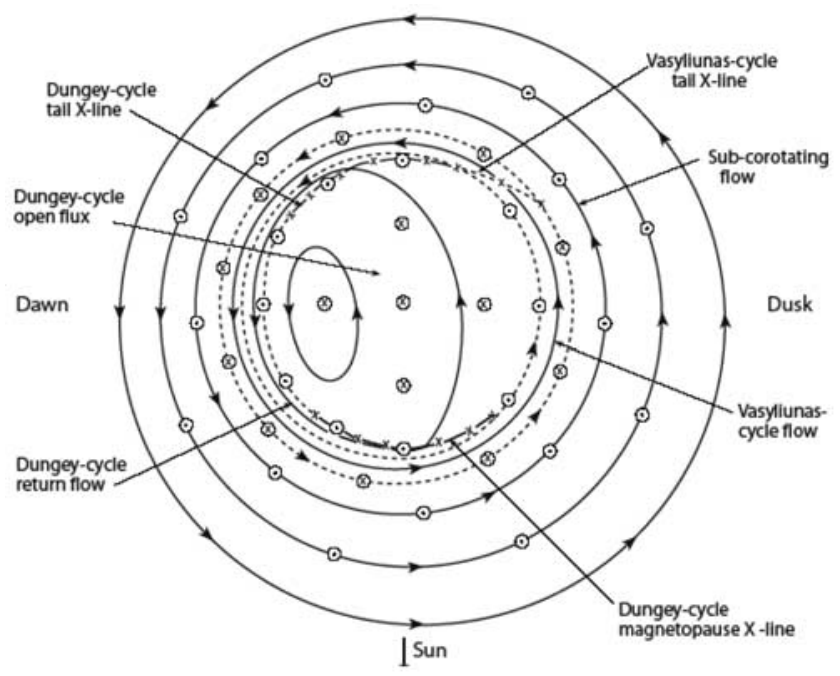

Figure 1. Polar view looking onto the northern ionosphere with the pole at the center, in a frame which is fixed relative to the Sun. The solid lines indicate plasma streamlines and the circled dots and crosses indicate regions of field-aligned current directed upward and downward, respectively. Taken from Cowley et al. [2004].

UVI imager on the Polar satellite during a period of northward IMF. They interpreted this feature as the signature of high-latitude reconnection and described its motion in response to IMF $B_{y}$ changes in coordination with observations of the large-scale convection flow. Frey et al. [2002] showed that the Lyman- $\alpha$ proton auroral brightness at the cusp footprint depends on the solar wind dynamic pressure. They also concluded that the cusp magnetic local time location depends on the IMF $B_{y}$ component, consistent with theoretical considerations of the partial penetration of the IMF $B_{y}$ into the magnetosphere [Cowley, 1981]. Fuselier et al. [2002] showed that the spot-like cusp signature located poleward of the oval merges with the dayside auroral oval as the IMF turns southward. These results were discussed in the context of antiparallel reconnection (at the high-latitude lobe magnetopause during northward IMF) and possible component reconnection (at the low-latitude magnetopause during southward IMF). Several studies demonstrated that the cusp footprint occurs at lower latitudes for southward IMF $B_{z}$ than for northward $B_{z}$ and described associated particle precipitation (latitude dispersion) and convection (shift of convection boundaries) characteristics [McCrea et al., 2000; Sandholt et al., 2001].

[4] Saturn's aurora has only recently been investigated with sufficient spatial and temporal resolution to address the question of the presence of an optical signature of the dayside cusp. Until far ultraviolet (FUV) observations with the Hubble Space Telescope became available, most information concerning the morphology of the FUV aurora was based on data collected with the Voyager UV spectrometer (UVS) during the Voyager 1 and 2 Saturn flybys. The aurora then appeared as a narrow ring at $80^{\circ}$ latitude region with no measurable emission inside the oval [Broadfoot et al., 1981; Sandel and Broadfoot, 1981]. At Saturn, as at Jupiter, both $\mathrm{HI}$ Lyman- $\alpha$ and $\mathrm{H}_{2}$ Lyman and Werner bands are present in the polar regions of both hemispheres. Saturn's aurora showed intensity temporal variations (factors of $\sim 2-5$ ) [Sandel et al., 1982], suggesting a solar wind controlled aurora. Outbursts of Lyman- $\alpha$ were intermittently observed with the International Ultraviolet Explorer (IUE) over a decade with a brightness of a few kiloRayleighs (kR) [Clarke et al., 1981; McGrath and Clarke, 1992], also not inconsistent with solar wind control of Saturn's aurora. The Faint Object camera on board the Hubble Space Telescope (HST) was used to obtain the first image of the north aurora [Gérard et al., 1995]. FUV images [Trauger et al., 1998] collected with the Wide Field Planetary Camera (WFPC2) showed a northern auroral arc appearing mostly fixed in local time near the dawn limb but with variable brightness. Images of the south aurora were obtained with the Space Telescope Imaging Spectrograph (STIS) between 11 October 1997 and 29 January 2004 [Gérard et al., 2004; Cowley et al., 2004; Clarke et al., 2005; Grodent et al., 2005]. They showed the presence of an auroral oval extending continuously from the midnight sector via dawn into the postnoon hours, though strongly varying in brightness with local time. The dayside main oval lies between $70^{\circ}$ and $80^{\circ}$ and is generally brighter and thinner in the morning than in the afternoon sector. The afternoon sector is characterized by more diffuse emission at higher latitudes. Weak emission is also observed poleward of the main oval up to the pole. A spiral structure of the main oval was occasionally seen. The brightness of the main oval ranges from below the STIS threshold of $1 \mathrm{kR}$ of $\mathrm{H}_{2}$ emission up to $\sim 75 \mathrm{kR}$. The total electron precipitated power varies between 20 and $140 \mathrm{GW}$, which is comparable to the Earth's active aurora but about two orders of magnitude less than on Jupiter. Comparison of spectra with a synthetic model of electron excited $\mathrm{H}_{2}$ were used to estimate the energy of the primary auroral electrons as $12 \pm 3 \mathrm{keV}$ [Gérard et al., 2004], based on a lowlatitude model atmosphere relying on Voyager occultation measurements.

[5] Cowley et al. [2004] have suggested that Saturn's aurora is associated with a ring of upward current flowing along the open-closed field line boundary generated by the difference in angular velocity between open and outer magnetosphere closed field lines. In Figure 1 we show a view of the flows and currents which are present in Saturn's ionosphere, associated with the three main flow regimes discussed previously by Hill [1979], Vasyliunas [1983], and Dungey [1961]. The three flow regions are as follows: (1) a lower-latitude region which maps to the subcorotating plasma in the equatorial plane, associated with a fieldaligned current system which is of insufficient magnitude and flowing at the wrong colatitude to account for the main oval auroras at Saturn [Cowley and Bunce, 2003], (2) a higher-latitude region of subcorotating flows where closed field lines are stretched out down-tail and eventually pinch off, forming a plasmoid, which is subsequently released downtail (the "Vasyliunas cycle"), and (3) a region of flow which is driven by reconnection at the dayside magnetopause in which "open" field lines mapping to the tail lobes flow antisunward over the poles, and following reconnection in the tail, return to the dayside, drawn here principally via dawn, in a single cell convection pattern (the "Dungey cycle"). It is the upward directed field-aligned current along the boundary between open and closed field lines (shown by the inner dashed circle), which is suggested to be associated 
with the main auroral oval at Saturn, possibly augmented by more diffuse emissions in the region immediately equatorward, due to hot plasma production and precipitation in the Dungey and Vasyliunas cycles [Cowley et al., 2005]. In this case, the main oval aurora is expected to be strongly modulated by the upstream solar wind conditions, as will be discussed in section 2 with respect to the coordinated Cassini-HST observations.

[6] Gérard et al. [2004] have suggested that dayside UV emissions in Saturn's polar ionosphere show observational evidence of the kronian "cusp" in the noon sector. They described the emission as presenting two distinct states. The first is a bright arc-like feature located in the prenoon sector that appears as an intensification of the main oval. The second is a more diffuse "spot" of aurora lying poleward of the general location of the main auroral oval, possibly related to different upstream interplanetary magnetic field (IMF) orientations. Bunce et al. [2005a] took up the suggestion that these emissions correspond to the cusp. They showed that direct precipitation of electrons in the cusp regions is not capable of producing significant UV aurora. Instead, they investigated the possibility that the UV emissions described by Gérard et al. [2004] are associated with reconnection occurring at the dayside magnetopause, possibly pulsed, analogous to flux transfer events seen at the Earth. As discussed for the case of the Jovian cusp by Bunce et al. [2004], pulsed reconnection at the low-latitude dayside magnetopause for the case of northward IMF will give rise to pulsed twin-vortical flows in the magnetosphere and ionosphere in the vicinity of the open-closed field-line boundary and hence to bipolar field-aligned currents centered in the vortical flows which close at one end in ionospheric Pedersen currents and at the other end in the Chapman-Ferraro currents on the magnetopause. In the case of southward IMF and high-latitude lobe reconnection, Bunce et al. [2005a] also expect that the vortical flows are displaced poleward of the open-closed field line boundary such that the field-aligned currents are also reversed compared with the former case. These perturbation currents associated with pulsed reconnection at the magnetopause will straddle (for the case of northward IMF) or sit slightly poleward of (for the case of southward IMF) the open-closed field line boundary associated with the main auroral oval. In addition to the variability in flows and currents expected to be present due to northsouth fluctuations in the IMF, modulation is also introduced by the large-scale structure of the solar wind and IMF at Saturn's orbit due to corotating interaction regions (CIRs) [Jackman et al., 2004]. Consequently, Bunce et al. [2005a] also considered a slow and a fast flow model to account for the highly developed nature of CIRs at Saturn's distance from the Sun, causing the solar wind and IMF to be separated into distinct regions of rarefaction and compression.

[7] During January 2004, a unique campaign took place during which magnetic field, plasma, and radio wave instruments on board the Cassini-Huygens spacecraft measured the in situ solar wind and embedded IMF while HST simultaneously observed the far ultraviolet aurora in Saturn's southern hemisphere. Results concerning the correlations between the main oval and the solar wind characteristics, the shape of the auroral region, its level of corotation, and brightness distribution were described by Clarke et al. [2005], Crary et al. [2005], Cowley et al. [2005], Bunce et al. [2005b], and Grodent et al. [2005]. The correlation between the auroral FUV emission and kilometric radio emission was analyzed by Kurth et al. [2005]. In this study we describe the main features of the FUV signature of cusp precipitation in these data and relate it to the characteristics of the IMF measured upstream with the Cassini orbiter en route to Saturn. We compare measured brightness with the energy fluxes expected from the model of Bunce et al. [2005a].

\section{Observations}

\subsection{HST Images}

[8] A total of 68 STIS images of Saturn's auroras, covering 17 HST orbits, were obtained with the photoncounting detector 25MAMA (Multi-Anode Micro channel Array) in the period between 8 and 30 January 2004 during the Cassini-HST campaign. The $24.7 \times 24.7 \operatorname{arcsec}^{2}$ field of view of STIS includes the full planet's disk and a fraction of the ring system. Two imaging modes were used for this data set. In the clear mode, the passband extends from 115 to $180 \mathrm{~nm}$ and is sensitive to the $\mathrm{H}_{2}$ Lyman and Werner bands as well as the strong $\mathrm{H}$ Lyman- $\alpha$ line. In the "filtered" mode, an $\mathrm{SrF}_{2}$ filter was added in the optical path, rejecting Lyman- $\alpha$. The MAMA array has $1024 \times$ 1024 pixels with a linear size of 0.0243 arcsec, thus providing a field of view of $24.7 \times 24.7 \operatorname{arcsec}^{2}$ with a 0.08 arcsec full width at half maximum point spread function. The effective spatial resolution projected at Saturn is thus approximately $500 \mathrm{~km}$. For a feature located at the central meridian longitude (CML) the smearing introduced by the planetary rotation is $0.56^{\circ}$ in longitude in $1 \mathrm{~min}$. The procedure used to determine the position of the center of the planet and the coordinates of each pixel was described by Gérard et al. [2004] and Grodent et al. [2005]. It is thus possible to generate polar (orthographic) projections. As part of the data reduction process, the images were flat-fielded using the most recent low-order flat field and pixel-to-pixel files provided by the Space Telescope Science Institute (STScI). Dark counts were subtracted using the appropriate STScI dark files. Maps were built in a system fixed relative to the central meridian, simulating an observer looking from above the north pole through the planet.

[9] Each orbit consists of four consecutive images. The first visit (V1) or "full rotation visit" comprised five consecutive HST orbits. The other visits (V2 to V13) all consist of one orbit approximately every 2 days. The clear images were accumulated for $270 \mathrm{~s}$ and the filtered images were obtained with exposure times varying from $640 \mathrm{~s}$ to 740 s. A first filtered image was followed by two "clear" exposures and another filtered image. The full list of observations was given by Grodent et al. [2005]. During this campaign, the sub-Earth planetocentric latitude changed from $25.65^{\circ}$ to $25.98^{\circ}$, and the subsolar longitude remained within $1^{\circ}$ of the sub-Earth longitude. Table 1 summarizes the times and CMLS of the 13 visits. The variations of the global morphology, brightness, and their relations with in situ solar wind measurements by Cassini were described by 
Clarke et al. [2005], Cowley et al. [2005], Bunce et al. [2005b], more detailed by Grodent et al. [2005], and are summarized hereafter.

\subsection{In Situ Solar Wind Measurements}

[10] During this campaign, the structure of the interplanetary medium was measured by the Cassini spacecraft en route to Saturn. During this period, Cassini was located $\sim 0.2$ AU upstream of Saturn in the radial direction and was off the planet-Sun line by $\sim 0.5$ AU toward dawn. Using an average solar wind speed of $\sim 500 \mathrm{~km} \mathrm{~s}^{-1}$, the radial propagation delay from Cassini to Saturn is $\sim 17$ hours [Crary et al., 2005]. Jackman et al. [2004] find that the data during the Cassini approach phase to Saturn show that the interplanetary magnetic field (IMF) structure generally consists of two sectors during each rotation of the Sun, with crossings of the heliospheric current sheet embedded within few-day high field compression regions, surrounded by several day rarefaction regions. This pattern is consistent with that expected to be generated by recurrent CIRs during the declining phase of the solar cycle. The top four panels of Figure 2 show the components of the magnetic field in the RTN (radial, tangential, normal) coordinate reference system and the total magnitude measured by the Cassini magnetometer during the interval 1-31 January 2004. RTN is a right-handed spherical polar system referenced to the Sun's spin axis, in which $B_{R}$ is positive radially outward from the Sun toward the spacecraft, $B_{T}$ is the azimuthal component positive in the direction of planetary motion, and $B_{N}$ is the component positive northward in the solar equatorial plane. Note that the orientation of Saturn's magnetic poles is reversed compared to the Earth's case. The fifth panel shows an estimate of the low-latitude dayside reconnection voltage (in units of $\mathrm{kV}$ ) across Saturn's magnetopause associated with the production of open flux based by analogy with the results of studies made at the Earth [Jackman et al., 2004]. In the estimate of Jackman et al. [2004], large low-latitude reconnection voltages are favored for northward pointing IMF at Saturn, reversed to the case for Earth due to the opposite sense of the planetary field. The expression for the voltage associated with open flux production is

$$
\Phi_{L-L}=V_{s w} B_{\perp} L_{0} \cos ^{4}\left(\frac{\theta}{2}\right),
$$

where $V_{\mathrm{sw}}$ is the radial speed of the solar wind, $B_{\perp}$ is the strength of the IMF component perpendicular to the radial flow, $L_{0}$ is a scale length assumed to be $10 R_{S}$ (where $1 R_{S}=$ $60228 \mathrm{~km}$ ) by analogy with the Earth, and theta is the clockangle of the IMF relative to Saturn's northern magnetic axis. The sixth panel in Figure 2 indicates an estimated voltage calculated for high-latitude (lobe) reconnection. In this case, large high-latitude reconnection voltages are associated with southward pointing IMF. This formula is given by

$$
\Phi_{H-L}=\frac{1}{2} V_{s w} B_{\perp} L_{0} \sin ^{4}\left(\frac{\theta}{2}\right),
$$

where the cos variation in the original northward IMF variation has been replaced with a sin dependence, thus producing large voltages for intervals of southward pointing IMF $B_{z}$. These two different scenarios will be referred to henceforth in this paper as the voltages associated with lowand high-latitude reconnection, respectively. The overall magnitude of the voltage associated with high-latitude reconnection is also estimated to be roughly half of that associated with low-latitude reconnection (see discussion by Bunce et al. [2005a]). These two parameters act together to indicate the level of dayside reconnection that may reasonably be expected to be present if the field which is measured at Cassini is the same as the field which impinges upon Saturn. However, as discussed above, the spacecraft is located at $\sim 0.5 \mathrm{AU}$ off the planet-Sun line (corresponding to $\sim 3^{\circ}$ of heliographic longitude). Therefore the field magnitude measured at Cassini is used here as an indication of the magnitude of the field present at Saturn, but the short timescale fluctuations in the $B_{N}$ component (i.e., IMF $B_{z}$ ) cannot be reliably propagated. We have thus overplotted a simplified "maximum" voltage (seen by the upper most black lines in each of panels 5 and 6), which simply estimates the reconnection voltage from the magnitude of the field assuming a purely northward or southward IMF direction in each case. It is these potentials which will now be discussed in the context of the HST observations. Using a 17-hour propagation delay, the HST visits are indicated in Figure 2 by the vertical dashed lines similarly marked V1 to 13. As discussed by Crary et al. [2005], the timing in this delay relative to the interplanetary data is unknown to within several hours, due to nonradial propagation effects and the separation of the spacecraft and the planet in heliographic longitude.

[11] The highly structured nature of the IMF was discussed in detail for this interval by Bunce et al. [2005b]. The HST observations took place in a period when different solar wind regimes may be distinguished. Visits V1-5 were made during a low-field rarefaction interval, which followed a major compression during 1-5 January. Here the total IMF strength is typically $<0.1 \mathrm{nT}$, the maximum lowlatitude dayside reconnection voltage (for a purely northward IMF as described above) is $\sim 20 \mathrm{kV}(\sim 10 \mathrm{kV}$ on average for the maximum high-latitude reconnection voltage), and the solar wind dynamic pressure (when available) is $\sim 0.003 \mathrm{nPa}$ [Crary et al., 2005]. HST images show a correspondingly "quiet" auroral oval, which remains throughout the low solar wind activity interval. Cowley et al. [2005] indicate that the decreasing size of the auroral oval over this time period is suggestive of a nightside reconnection rate stronger than the dayside rate, thus closing flux at a greater rate than it can be opened.

[12] Visits V6-10 took place after a "minor" compression event on 17 January, when the field strength was typically $0.2-0.4 \mathrm{nT}$, the maximum low-latitude dayside reconnection voltage was $\sim 50-100 \mathrm{kV}$, and the solar wind dynamic pressure was $\sim 0.01 \mathrm{nPa}$. The solar wind density was $\sim 0.03 \mathrm{~cm}^{-3}$, the solar wind velocity was decreasing, and the reconnection voltages were correspondingly "medium" in strength. The maximum high-latitude reconnection voltage estimate gives on average $40 \mathrm{kV}$, with peaks of up to $70 \mathrm{kV}$. During this period, the oval is first contracted between V5 and 6 and is significantly brighter than the previous five images and appears to form a spiral. Cowley et $a l$. [2005] suggested that this contraction of the auroral oval and enhanced precipitation are indicative of an episode of strong tail reconnection and the closure of open flux in the 


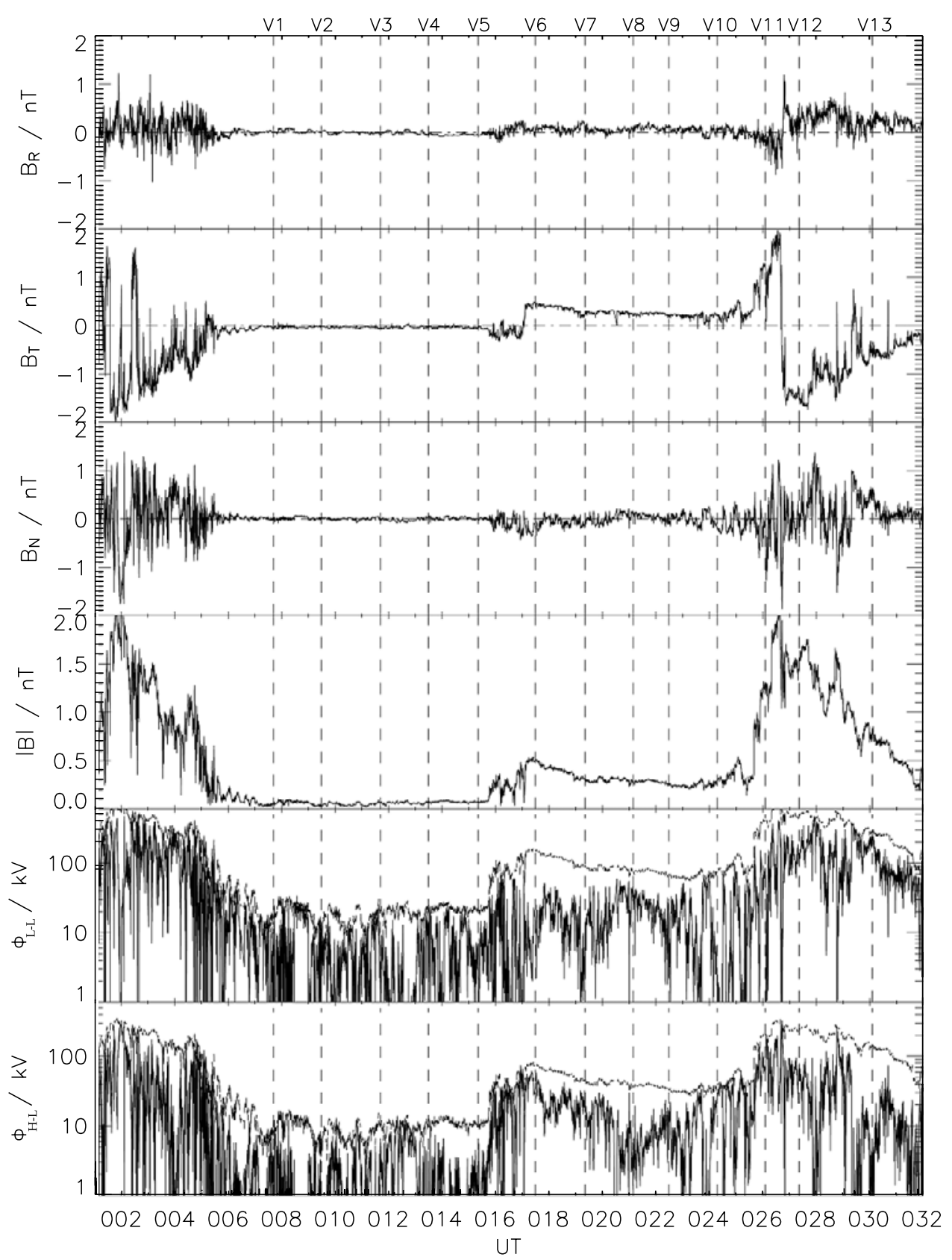

Figure 2. Plot of the Cassini magnetic field measurements. The first four panels show the components of the magnetic field in RTN coordinates $B_{R}, B_{T}, B_{N}$, and the magnitude of the field $|\mathrm{B}|$ in units of $\mathrm{nT}$. The fifth and sixth panels show the estimated reconnection voltage in units of $\mathrm{kV}$, derived from the magnetic field and the velocity data as described in the text. The high-frequency trace shows the electric potential $(\mathrm{kV})$ calculated for low-latitude dayside reconnection and high-latitude reconnection in panels 5 and 6 respectively. The uppermost smooth lines in each panel indicate a simple "maximum" voltage in each case, as described in the text. The dashed vertical lines indicate the approximate times of the HST images, shifted by 17 hours. Adapted from Bunce et al. [2005b].

tail. During the "medium" activity interval V6-10, the oval slowly expands, indicating open flux is added on the dayside at a faster rate than was being closed on the nightside.

[13] Finally, visits V11-13 took place just following the start of a major compression period observed during $25-$ 30 January, when the field strength increased to $\sim 0.5-$ $2.0 \mathrm{nT}$, the estimated maximum low-latitude dayside reconnection voltage was typically $\sim 400 \mathrm{kV}(\sim 200 \mathrm{kV}$ for the estimate of the maximum high-latitude reconnection voltage), and the solar wind dynamic pressure was on average increased to $\sim 0.03 \mathrm{nPa}$. In this final period of HST observations, very dynamic and bright auroral emission was observed. This interval coincides with the major compression region that began on 25 January with the forward shock wave marking the start of the CIR compres- 

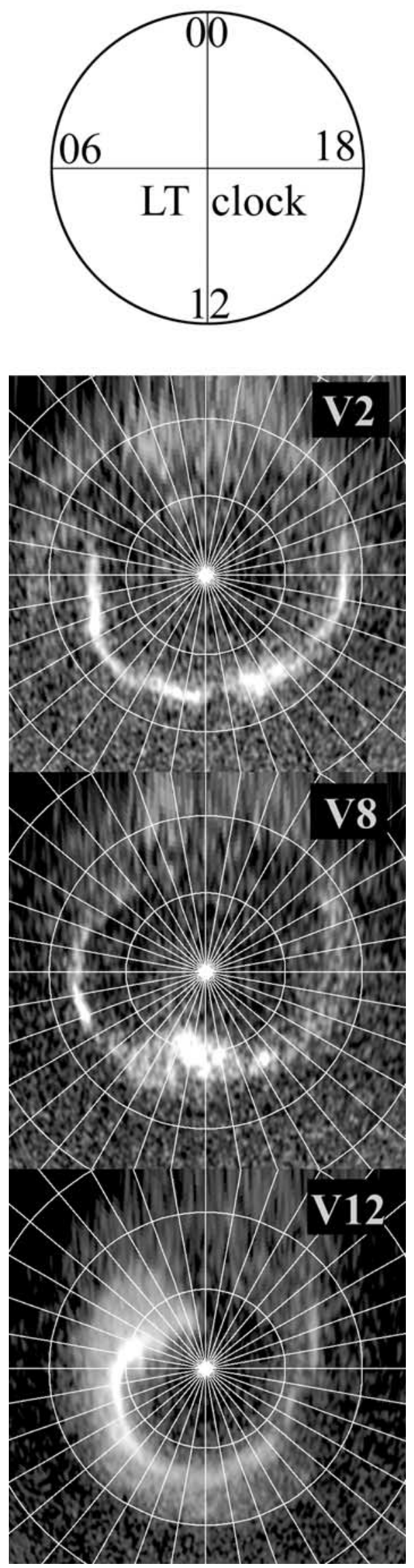

sion region. Images obtained on V11 show that the dark polar cap has been filled in with bright aurora. According to Cowley et al. [2005], this is an early effect of the onset of tail reconnections triggered by the sudden compression of the magnetosphere. Following this, V13 showed a bright, expanding oval.

[14] In summary, one can distinguish four time intervals over the period of coordinated Cassini-HST observations: a low-field rarefaction region from 8 to 16 January following a major compression event, a "minor" compression event on 17 January, a rarefaction region with intermediate field strengths from 19 to 25 January, and a major compression region from 26 to 30 January. The general morphology of the main oval can be understood in terms of the characteristics of the solar wind at Saturn and the balance between the rate of dayside IMF reconnection and nightside field line reconnection.

\section{Cusp-Solar Wind Relationship}

[15] Enhanced precipitation in the noon sector is not necessarily a signature of the dayside cusp. The sequence of observations performed during V1 shows that "blobs" of plasma carried by convection may easily be confused with the cusp enhancement if the time evolution of the global morphology is not available. We thus set criteria to discriminate between the two situations. A first condition is that the cusp emission must remain nearly fixed in local time over the $\sim 45$ min of observing time of an HST orbit. Instead, plasma "blobs" are observed to move eastward at $\sim 70 \%$ of the corotation angular velocity. Since four STIS images were collected during each HST orbit, the azimuthal eastward motion is expected to be $\sim 15^{\circ}$ over the $35 \mathrm{~min}$ separating the first and the last images of each orbit. Therefore we only consider cases when the noon sector enhancement moves eastward by less than $5^{\circ}$. We also set a brightness threshold on the identification of cusp features. They must clearly exceed the background level by at least a factor of two.

[16] Figure 3 shows examples of filtered STIS images collected at three different periods of the January 2004 campaign. The first image (Figure 3, V2) was obtained on 10 January (V2). At this time, the IMF magnitude was $\sim 0.05 \mathrm{nT}$ and $B_{N}$ was very small. Both the maximum lowand high-latitude reconnection potentials were below $20 \mathrm{kV}$.

Figure 3. Polar (orthographic) projections of HST-STIS images of Saturn's south FUV aurora. Images were taken on (top) 10 January (V2), (middle) 21 January (V8), and (bottom) 28 January (V12) 2004 and belong to the low-field rarefaction period, the recovery period following the 15 January solar wind compression, and the recovery period following the 25 January interplanetary shock, respectively. The image has been projected on a polar view where the south is seen by a virtual observer above the north pole, looking through the planet. In these viewing conditions, the south pole is at the center, the direction of the Sun (1200 LT) is conveniently oriented towards the bottom, dawn (0600 LT) to the left, dusk (1800 LT) to the right as indicated in the LT clock panel. The parallels are separated by $10^{\circ}$ of latitude and the meridians by $10^{\circ}$ of longitude. See color version of this figure at back of this issue. 


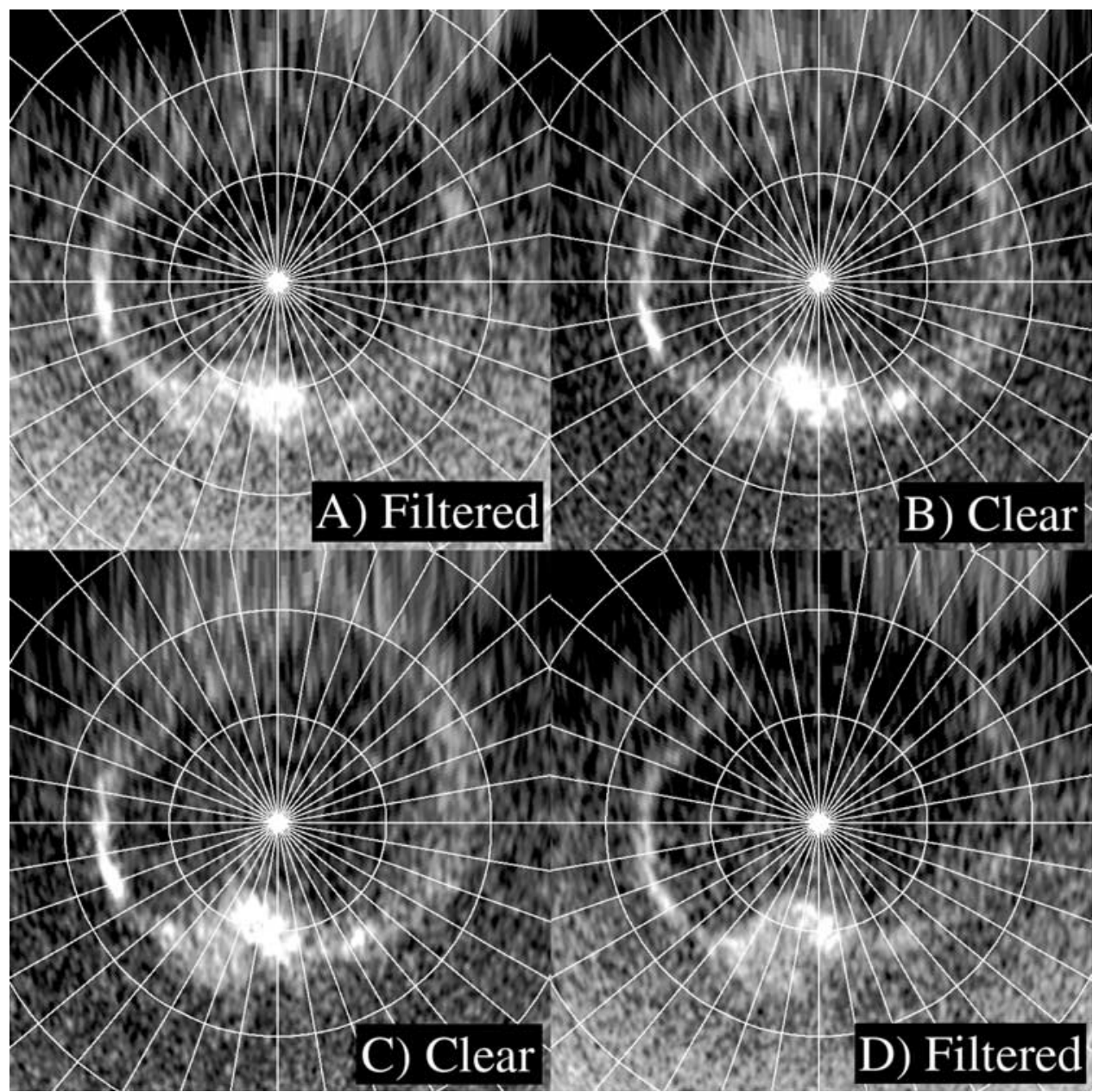

Figure 4. Polar projections of HST-STIS images of Saturn's southern hemisphere UV aurora taken on 21 January 2004 (Visit 8). (a) and (d) Images were taken in the SrF2 'filtered' mode; (b) and (c) images were unfiltered. See color version of this figure at back of this issue.

Solar wind conditions were thus inappropriate to produce significant dayside reconnection and cusp precipitation. The auroral oval is wide, which is characteristic of steady state solar wind conditions encountered during rarefaction periods (large open field line flux in the polar cap, no dayside reconnection). As expected, no enhancement is observed along the oval or poleward in this case. Figure 3 (V8) shows an image collected on 21 January (V8). Unlike the previous case, it shows a significant enhancement located near 1200 LT poleward of the main auroral oval. This cusp spot is comparatively as bright as the dawn sector oval where limb brightening probably increases the vertical intensity relative to features in the noon sector. This time period corresponds to the interval following the minor compression in the solar wind when the IMF intensity was about $0.5 \mathrm{nT}$ with the field mostly directed in the sense of positive $B_{t}$ (negative $B_{y}$ ) with fluctuations of the north-south component on short timescales. The maximum reconnection voltages at this approximate time for low-latitude and high-latitude reconnection are $\sim 80 \mathrm{kV}$ and $\sim 40 \mathrm{kV}$, respectively, the former being associated with the largest intensities for any of the HST images collected during the campaign, with the exception of V11, 12, and 13 when the enhancement of the main oval and the perturbed morphology masks out any signature of cusp emission The location of this cusp emission poleward of the main auroral oval is thus consistent with an interval of southward IMF and high-latitude reconnection. The full set of four STIS images collected during V8 is shown in Figure 4. The first filtered image is identical to Figure $3 \mathrm{~b}$. The scales of the first and fourth images have been adjusted to account for the higher sensitivity of the clear mode compared to the other two images with the $\mathrm{SrF}_{2}$ filter. The images, together with the coordinates of the spot in the noon sector given in Table 1, indicate that the spot center is consistently poleward of the main oval location and quasi-stationary in local time. The oval at this time exhibits a spiral structure indicative of active tail reconnection at a rate exceeding that of dayside reconnection. Finally, the image in Figure 3 (V12) was taken on 28 January (Visit 12), following the start of the major compression. The oval has a significantly lower size than previously, but it is also significantly thicker, especially in the morning and noon sectors. No cusp emission is observed poleward of the main oval in this case.

[17] In summary, a clear signature of cusp precipitation poleward of the oval is observed following the minor compression period when the maximum high-latitude reconnection voltage is a few tens of $\mathrm{kV}$ and is not obscured by other dynamical dominant processes. Images collected during V7 
Table 1. Time and Position of Near-Noon Auroral Brightening

\begin{tabular}{|c|c|c|c|c|c|c|c|c|c|}
\hline Visit & File & Date (January 2004) & Subsolar Long., ${ }^{\text {deg }}$ & Time, UT & CML & Lat., deg & Long., deg & Average UV, kR & UV Power, GW \\
\hline V1 & o8wi02c69 & 8 & $38.8-271.3$ & 0442 & 49.2 & - & - & - & \\
\hline $\mathrm{V} 2$ & o8wi01s19 & 10 & $219.5-236.9$ & 0441 & 230.1 & - & - & - & \\
\hline V3 & o8wi03dsq & 12 & $202.8-220.2$ & 0929 & 213.7 & -76.1 & 170 & 50 & 6.5 \\
\hline V4 & o8wi04rtq & 14 & $221.8-239.8$ & 0441 & 233.5 & -75.9 & 177 & 23 & 4.3 \\
\hline V5 & o8wi05h $2 q$ & 16 & $295.0-312.9$ & 0129 & 306.9 & -77.9 & 190 & 9 & 2.0 \\
\hline V6 & o8wi06baq & 18 & $224.2-242.2$ & 0440 & 236.4 & - & - & - & - \\
\hline V7 & o8wi07k0q & 20 & $297.7-315.7$ & 0128 & 310.1 & $-79.2^{\mathrm{b}}$ & 177 & 14 & 2.1 \\
\hline V8 & o8wi08b5q & 21 & $316.9-334.9$ & 2040 & 329.5 & $-79.8^{\mathrm{b}}$ & 175 & 21 & 5.0 \\
\hline V9 & o8wi09moq & 23 & $317.4-335.4$ & 0439 & 330.2 & -76.6 & 180 & 20 & 2.5 \\
\hline V10 & o8wi10bdq & 24 & $336.5-354.5$ & 2351 & 349.5 & -76.5 & 160 & 29 & 2.5 \\
\hline V11 & o8willhuq & 26 & $355.5-13.5$ & 1903 & 8.7 & - & - & - & \\
\hline V12 & o8wi12qwq & 28 & $302.9-320.9$ & 0127 & 316.3 & - & - & - & \\
\hline$\underline{V} 13$ & o8wil3llq & 30 & $357.7-15.6$ & 1901 & 11.3 & - & - & - & \\
\hline
\end{tabular}

${ }^{\mathrm{a}}$ Subsolar latitudes range from $-25.45^{\circ}$ to $-25.57^{\circ}$.

${ }^{\mathrm{b}}$ Indicates the presence of a cusp feature detached from the main oval.

(19-20 January) also exhibit a cusp-like signature (Figure 5) fixed in local time. However, the filtered images show that in this case, the noon feature is more elongated (along a meridian $\sim 5^{\circ}$ poleward of the oval) than in V8. An intensity gap is clearly observed at $\sim 1100$ LT. The cusp arc remains poleward of the morning sector location of the main oval and slowly fades out until it disappears at dusk. As mentioned before, cusp signatures at the latitude of or close to the main oval are more difficult to visually identify, as they may be local enhancements of the oval connected to subcororating plasma blobs. An example is shown in Figure 6 illustrating observations performed during V9. In this series, the intensification of the oval observed near 1200 LT is quasi-stationary. At this time, the IMF intensity was about $0.4 \mathrm{nT}$ and was directed nearly in the ecliptic plane. We now compare the cusp emission identified in Figures 4

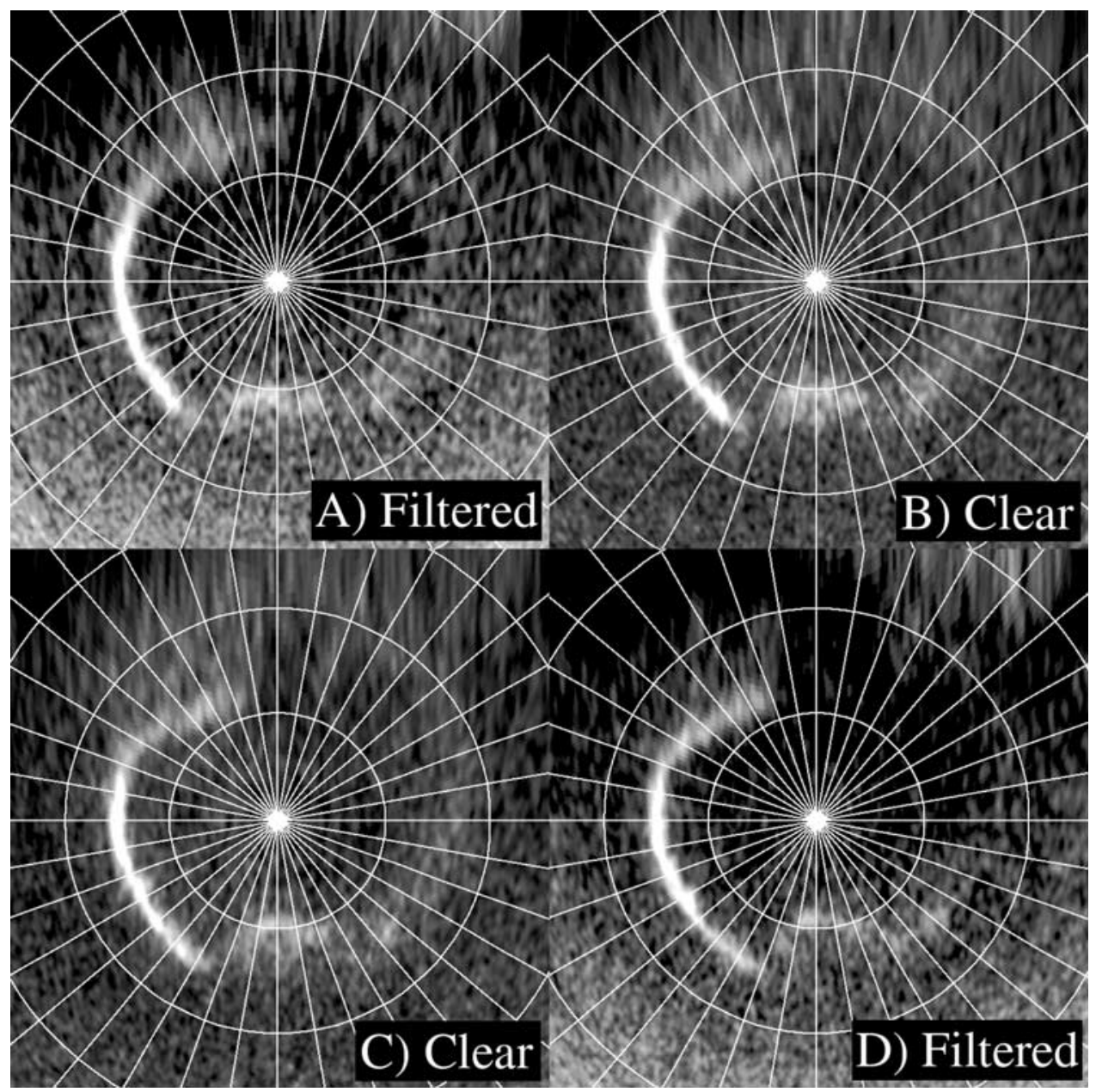

Figure 5. Polar projections of HST-STIS images of Saturn's southern hemisphere UV aurora taken on 20 January 2004 (Visit 7). See color version of this figure at back of this issue. 


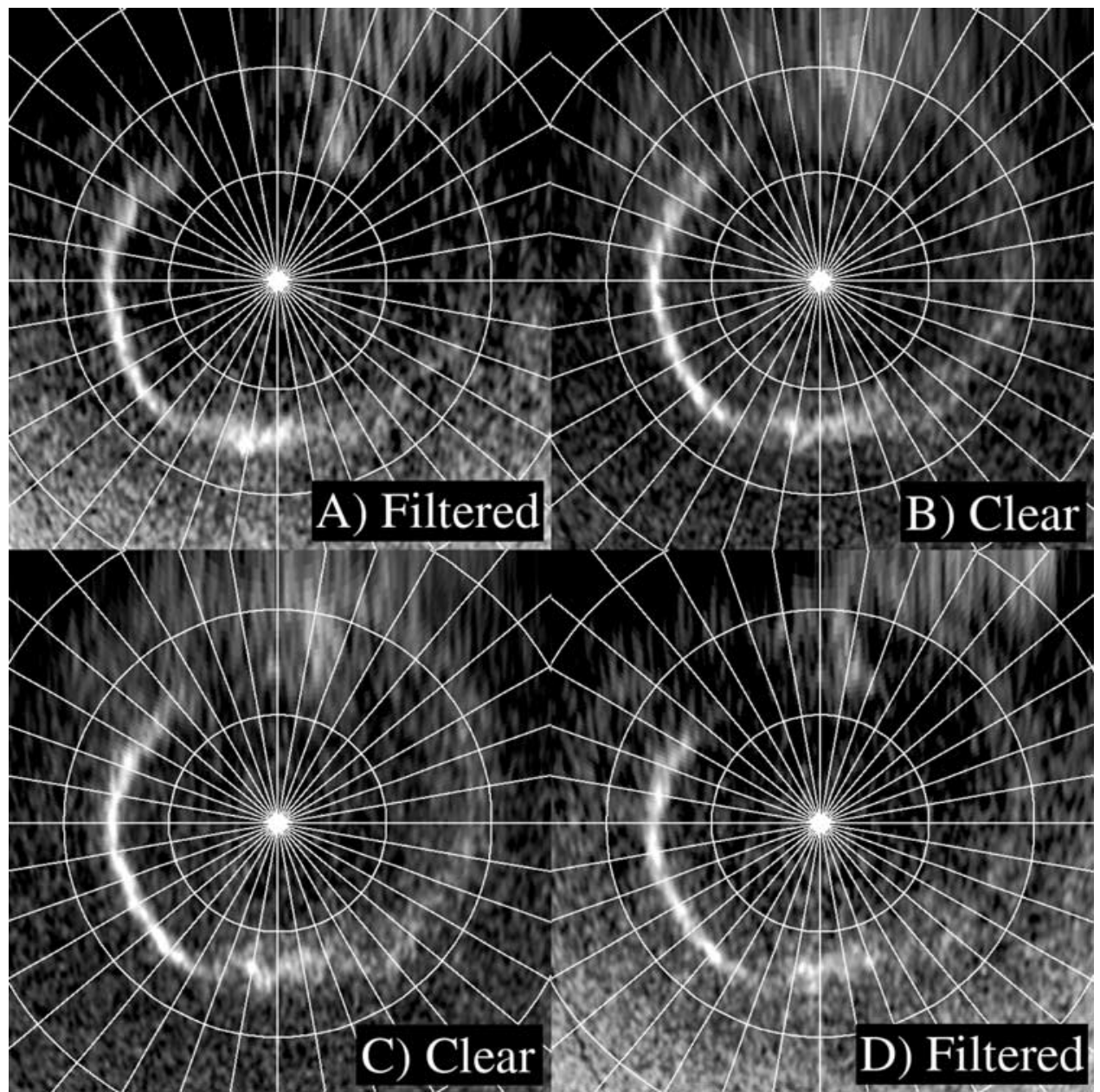

Figure 6. Polar projections of HST-STIS images of Saturn's southern hemisphere UV aurora taken on 23 January 2004 (Visit 9). See color version of this figure at back of this issue.

and 6 (V8 and 9) with the theoretical model of low- and high-latitude dayside reconnection presented by Bunce et al. [2005a].

\section{Comparison of FUV Brightness With the Cusp Model}

[18] As described in the introduction, Bunce et al. [2005a] have recently developed a conceptual model of the vortical flows and currents which arise due to reconnection, possibly pulsed, on the dayside magnetopause at Saturn. Here we now compare the HST-STIS observations of the candidate dayside cusp aurora described in the previous section, with the upstream in situ interplanetary medium sampled by the Cassini spacecraft en route to Saturn. In order to augment our understanding of Saturn's cusp processes, we now compare the UV observations made by the HST with the cusp model of Bunce et al. [2005a], which is appropriate to the solar wind conditions measured during the January interval.

[19] We will compare the two examples of what we understand to be dayside cusp related emission described in the previous section and shown in Figures 4 and 6, namely those identified on 22 January (V8) and 23 January (V9), with the theoretical model of Bunce et al. [2005a]. V8 is thought to be an example of a highlatitude "spot" indicative of southward IMF $B_{z}$. As discussed in the previous section, these data were collected during the interval of "intermediate" field strength, following the minor compression region that passed the spacecraft on 16 January. This level of intermediate field strength (which is on average $\sim 0.5 \mathrm{nT}$ ) was modeled by Bunce et al. [2005a] as being their "slow flow" model. As recently discussed by Bunce et al. [2004, 2005a], pulsed reconnection at the low-latitude dayside magnetopause for the case of northward IMF will give rise to pulsed twin-vortical flows in the magnetosphere and ionosphere in the vicinity of the open-closed field-line boundary (shown by the inner dashed circle in Figure 1) and hence to bipolar field-aligned currents centered in the vortical flows which close at one end in ionospheric Pedersen currents and at the other in the ChapmanFerraro currents on the magnetopause. In the case of high-latitude lobe reconnection for southward IMF, we also expect to have pulsed twin-vortical flows and corresponding bipolar field-aligned currents. However, the vortical flows in this case are displaced poleward of the open-closed field line boundary and are reversed in sense, such that the field-aligned currents are also reversed compared with the former case. The upward 
Table 2. Predicted Cusp Parameters Using the Conceptual Model of Bunce et al. [2005a]

\begin{tabular}{|c|c|c|c|c|c|c|c|}
\hline & & Flow Factor $F^{b}$ & Voltage, ${ }^{\mathrm{c}} \mathrm{kV}$ & Ave $\mathrm{FAC},{ }^{\mathrm{d}} \mu \mathrm{A} \mathrm{m}{ }^{-2}$ & Total Current, ${ }^{\mathrm{e}} \mathrm{MA}$ & Average $\mathrm{UV},{ }^{\mathrm{f}} \mathrm{kR}$ & UV Power, ${ }^{g}$ GW \\
\hline \multirow[t]{4}{*}{ Visit 8} & Area $^{\mathrm{a}} 8 \times 10^{12} \mathrm{~m}^{2}$ & 1 & $\sim 59$ & $\sim 0.007$ & $\sim 0.05$ & $0.7(0.07)$ & $\sim 0.06$ \\
\hline & & 2 & $\sim 118$ & $\sim 0.013$ & $\sim 0.11$ & $2.8(0.3)$ & $\sim 0.24$ \\
\hline & & 4 & $\sim 238$ & $\sim 0.027$ & $\sim 0.21$ & $11.3(1.1)$ & $\sim 1,0$ \\
\hline & & 6 & $\sim 358$ & $\sim 0.040$ & $\sim 0.32$ & $25.4(2.5)$ & $\sim 2.2$ \\
\hline \multirow[t]{4}{*}{ Visit 9} & Area $6 \times 10^{12} \mathrm{~m}^{2}$ & 1 & $\sim 110$ & $\sim 0.127$ & $\sim 0.80$ & $18.4(2.7)$ & $\sim 1.3$ \\
\hline & & 2 & $\sim 222$ & $\sim 0.151$ & $\sim 0.95$ & $28.9(4.2)$ & $\sim 2.1$ \\
\hline & & 4 & $\sim 446$ & $\sim 0.199$ & $\sim 1.26$ & $60.4(8.7)$ & $\sim 4.3$ \\
\hline & & 6 & $\sim 668$ & $\sim 0.248$ & $\sim 1.56$ & $105.5(15.2)$ & $\sim 7.6$ \\
\hline
\end{tabular}

${ }^{a}$ The total area, equatorward and poleward of the merging gap, considered in the model calculation.

${ }^{\mathrm{b}}$ The factor by which the vortical flow speeds, $\mathrm{V}_{2}$ are increased.

${ }^{\mathrm{c}}$ The implied voltage required to increase the vortical flow speeds by the factor given in footnote $\mathrm{b}$.

${ }^{\mathrm{d}}$ The magnitude of the field-aligned current density averaged over the area, obtained from the total current divided by the area defined in footnote a.

${ }^{\mathrm{e}}$ The total upward directed field-aligned current integrated over the area defined in footnote a. Any small region of downward current in the area is not included.

${ }^{\mathrm{f}}$ The averaged emission rate in the FUV (B and $\mathrm{C}$ singlet $\mathrm{H}_{2}$ states), obtained from the averaged precipitating energy flux. The values in brackets indicate the subsolar or high-latitude sheath plasma values in the IMF $B_{z}>0$ and IMF $B_{z}<0$ models, respectively.

${ }^{\mathrm{g}}$ The total power emitted in the FUV from the area given in footnote a, obtained as $20 \%$ of the total precipitating particle power.

currents which lie along the open-closed field line boundary are thought to be responsible for the production of the main auroral oval at Saturn [Cowley et al., 2004]. For the case of northward IMF $B_{z}$ the pulsed currents will modulate the general upward field-aligned current around the boundary, while for the case of southward IMF the pulsed currents are poleward of the main auroral oval currents. Bunce et al. [2005a] have also modeled the azimuthal asymmetries associated with $\operatorname{IMF} B_{y}$, but this will not be addressed in this paper.

[20] In Table 2 we show the results from the (modified) model of Bunce et al. [2005a]. In each case (V8 and 9) we start with the "slow model" which, as discussed by Bunce et al. [2005a] is intended to represent intermediate IMF strengths observed at this time. In the model the vortical flows associated with reconnection are taken to be elliptical in form, and centered at either end of the merging gap. The parameter V2 (in their equation (8a)) represents the strength of the vortical flows and has then been chosen such that the total voltage along the merging gap associated with the flow is $\sim 100 \mathrm{kV}$ in the case of northward IMF $B_{z}$ and $\sim 50 \mathrm{kV}$ in the case of southward IMF $B_{z}$. We have simply modified the strength of the vortices here by introducing a flow factor $F=1,2,4,6$, to match the UV brightness and power with that measured during V8 and 9. The second column indicates the voltage $(\mathrm{kV})$ associated with the vortices augmented by the factors given in the first column. The voltages shown in Table 2 can be compared with those we have estimated based on the Cassini interplanetary data shown in Figure 2. The third column shows the averaged fieldaligned current density over the emission area (see below), and the fifth and sixth columns give the averaged UV brightness (kR) and the total power output (GW). The areas in the model are chosen by looking at the field-aligned current density contours in the model that are above a simple background level $\left(\sim 0.05 \mu \mathrm{A} \mathrm{m}^{-2}\right)$, over which we integrate to derive the parameters in Table 2. For the high-latitude case (V8), in the southward IMF $B_{z}$ model, we obtain an area of $4 \times 10^{12} \mathrm{~m}^{2}$ for both the plasma mantle region (the high-latitude lobe population equatorward of the merging gap containing plasma originating from the magnetosheath), and the same area in the high- latitude magnetosheath region (the population poleward of the merging gap) giving a total area of $8 \times 10^{12} \mathrm{~m}^{2}$. For the case of the subsolar reconnection (V9) we consider a total area of $6 \times 10^{12} \mathrm{~m}^{2}$. This is divided evenly between the region equatorward of the open-closed field line boundary, in the magnetospheric plasma regime, and the region poleward of the open-closed field line boundary in the magnetosheath plasma regime.

[21] As indicated in Table 2, for V8, a factor of $\sim 6$ in the slow model is required to produce an average UV brightness of $\sim 26 \mathrm{kR}$ and a total UV power of $\sim 2.25 \mathrm{GW}$, to be compared with the HST observed values of $21 \mathrm{kR}$ and $5 \mathrm{GW}$. However, this requires a voltage value of $>200 \mathrm{kV}$, which is approximately a factor of 4 higher than the maximum high-latitude reconnection voltage estimated at this time (and is also the same as the standard slow model). For V9, the model agrees well and we obtain an average UV brightness of $\sim 18 \mathrm{kR}$ and a total UV power of $\sim 1.3 \mathrm{GW}$ using the standard slow model (where flow factor $F=1$ ). This compares to the HST observed values of $20 \mathrm{kR}$ and 2.5 GW. The standard slow model of Bunce et al. [2005a] for the case of northward IMF requires peaks of $\sim 100 \mathrm{kV}$, in fair agreement with the maximum low-latitude reconnection voltage during V9. Overall, we find that the conceptual model of Bunce et al. [2005a] provides a reasonable estimate of the UV brightness and power for the two examples of UV cusp emission presented here. However, the model agrees significantly better for the case indicative of low-latitude reconnection than for the case thought to be associated with high-latitude reconnection.

[22] The question whether the reconnection is steady or pulsed is not a significant factor in this study, as the model represents a snapshot of the flow and current at a particular time. It can in principle represent a steady situation or one that is evolving. For low-latitude reconnection the pattern is probably part of an evolving pattern at an early phase of a burst when the flow is reasonably localized around the merging gap and before it is spread over the whole polar region. The assumption of a pulsed flow in this context also allows to increase the instantaneous voltage to some factor larger than the expected mean value. However, for high-latitude reconnection, one 
might reasonably expect a more compact flow system poleward of the open-closed field line boundary anyway, so whether the instantaneous flow pattern analyzed is part of an evolving pulsed system or a more steady situation is difficult to determine.

\section{Conclusion}

[23] Observations of Saturn's FUV south aurora were made with the Hubble Space Telescope in parallel with in situ measurements of the solar wind plasma characteristics and embedded interplanetary magnetic field as the Cassini probe was approaching Saturn. The IMF was highly structured during this interval. The dayside reconnection voltage across Saturn's magnetopause associated with the production of open flux and lobe reconnection is calculated from the solar wind IMF and speed by analogy with the scenario at Earth. A dayside FUV signature of intense electron precipitation similar to that previously identified by Gérard et al. [2004] is found poleward of or along the main oval following the minor compression period when the IMF intensity was a few tenths of nT and the potential rapidly varied in the range $30-100 \mathrm{kV}$. Uncertainties on the time delay between the HST images and the arrival of the solar wind plasma at Saturn's magnetopause prevent accurate relative time positioning of aurora and the solar wind changes. However, these results are in good general agreement with the conceptual model by Bunce et al. [2005a] where pulsed reconnection at the low-latitude dayside magnetopause is expected to give rise to pulsed twin-vortical flows in the magnetosphere and hence to bipolar field-aligned currents centered in the vortical flows when the IMF is directed northward. In the case of southward IMF and high-latitude lobe reconnection, the model predicts that the vortical flows are displaced poleward of the open-closed field line boundary with reversed field-aligned currents compared with the former case. The slow model gives good agreement in the case of lowlatitude reconnection, assumed to be present during intervals of northward IMF $B_{z}$ but does not well reproduce the FUV brightness and power for high-latitude lobe reconnection, assumed to be present during intervals of southward IMF $B_{z}$. This indicates that lobe reconnection is perhaps more efficient than Bunce et al. [2005a] have assumed or that other mechanisms may be present. Further observations and modeling work will be required to improve our understanding of this complex system.

[24] Acknowledgments. This work is based on observations with the NASA/ESA Hubble Space Telescope, obtained at the Space Telescope Science Institute (STScI), which is operated by the AURA, Inc. for NASA. JCG and DG acknowledge support from the Belgian Fund for Scientific Research (FNRS). The PRODEX program of ESA provided financial support for this research to the University of Liège. This research was also funded by grants from the Space Telescope Science Institute and from NASA to the University of Michigan. SWHC was supported by PPARC Senior Fellowship PPA/N/S/2000/00197.

[25] Arthur Richmond thanks Harald Frey and George Gladstone for their assistance in evaluating this paper.

\section{References}

Broadfoot, A. L., et al. (1981), Extreme ultraviolet observations from Voyager 1 encounter with Saturn, Science, 212, 206.
Bunce, E. J., S. W. H. Cowley, and T. K. Yeoman (2004), Jovian cusp processes: Implications for the polar aurora, J. Geophys. Res., 109, A09S13, doi:10.1029/2003JA010280.

Bunce, E. J., S. W. H. Cowley, and S. E. Milan (2005a), Interplanetary magnetic field control of Saturn's polar cusp aurora, Ann. Geophys, 23, 1405 .

Bunce, E. J., S. W. H. Cowley, C. M. Jackman, J. T. Clarke, F. J. Crary, and M. K. Dougherty (2005b), Cassini observations of the interplanetary medium upstream of Saturn and their relation to the Hubble Space Telescope aurora data, Adv. Space Res., in press.

Clarke, J. T., H. W. Moos, S. K. Atreya, and A. L. Lane (1981), IUE detection of bursts of H Ly a emission from Saturn, Nature, 290, 226.

Clarke, J. T., et al. (2005), Morphological differences between Saturn's ultraviolet aurorae and those of Earth and Saturn, Nature, $433,717$.

Cowley, S. W. H. (1981), Magnetospheric asymmetries associated with the Y-component of the IMF, Planet. Space Sci., 29, 79.

Cowley, S. W. H., and E. J. Bunce (2003), Corotation-driven magnetosphere-ionosphere coupling currents in Saturn's magnetosphere and their relation to the auroras, Ann. Geophys., 21, 1691.

Cowley, S. W. H., E. J. Bunce, and R. Prangé (2004), Saturn's polar ionospheric flows and their relation to the main auroral oval, Ann. Geophys., 22, 1379 .

Cowley, S. W. H., S. V. Badman, E. J. Bunce, J. T. Clarke, J.-C. Gérard, D. Grodent, C. M. Jackman, S. E. Milan, and T. K. Yeoman (2005), Reconnection in a rotation-dominated magnetosphere and its relation to Saturn's auroral dynamics, J. Geophys. Res., 110, A02201, doi:10.1029/ 2004JA010796.

Crary, F. J., et al. (2005), Solar wind dynamic pressure and electric field as the main factors controlling Saturn's aurorae, Nature, 433, 720

Dungey, J. W. (1961), Interplanetary field and the auroral zones, Phys. Rev. Lett., 6, 47.

Frey, H. U., S. B. Mende, T. J. Immel, S. A. Fuselier, E. S. Claflin, J.-C. Gérard, and B. Hubert (2002), Proton aurora in the cusp, J. Geophys. Res., 107(A7), 1091, doi:10.1029/2001JA900161.

Fuselier, S. A., B. J. Anderson, and T. G. Onsager (1997), Electron and ion signatures of field line topology at the low-shear magnetopause, J. Geophys. Res., 102, 4847.

Fuselier, S. A., H. U. Frey, K. J. Trattner, S. B. Mende, and J. L. Burch (2002), Cusp aurora dependence on IMF Bz, J. Geophys. Res., 107(A7), 1111, doi:10.1029/2001JA900165.

Gérard, J.-C., V. Dols, D. Grodent, J. H. Waite, G. R. Gladstone, and R. Prangé (1995), Simultaneous observations of the saturnian aurora and polar haze with the HST/FOC, Geophys. Res. Lett., 22, 2685.

Gérard, J.-C., D. Grodent, J. Gustin, A. Saglam, J. T. Clarke, and J. T. Trauger (2004), Characteristics of Saturn's FUV aurora observed with the Space Telescope Imaging Spectrograph, J. Geophys. Res., 109, A09207, doi:10.1029/2004JA010513.

Grodent, D., J.-C. Gérard, S. W. H. Cowley, E. J. Bunce, and J. T. Clarke (2005), Variable morphology of Saturn's southern ultraviolet aurora, J. Geophys. Res., 110, A07215, doi:10.1029/2004JA010983.

Hill, T. W. (1979), Inertial limit on corotation, J. Geophys. Res., 84, 6554. Jackman, C. M., N. Achilleos, E. J. Bunce, S. W. H. Cowley, M. K. Dougherty, G. H. Jones, and S. E. Milan (2004), Interplanetary magnetic field conditions at $\sim 9$ A.U. during the declining phase of the solar cycle and its implications for Saturn's magnetospheric dynamics, J. Geophys. Res., 109, A11203, doi:10.1029/2004JA010614.

Kurth, W. S., et al. (2005), An Earth-like correspondence between Saturn's auroral features and radio emission, Nature, 433, 722.

McCrea, I. W., M. Lockwood, J. Moen, F. Pitout, P. Eglitis, A. D. Aylward, J.-C. Cerisier, A. Thorolfssen, and S. E. Milan (2000), ESR and EISCAT observations of the response of the cusp and cleft to IMF orientation changes, Ann. Geophys., 18, 1009.

McGrath, M. A., and J. T. Clarke (1992), H I Lyman alpha emission from Saturn (1980-1990), J. Geophys. Res., 103, 20,237.

Milan, S. E., M. Lester, S. W. H. Cowley, and M. Brittnacher (2000), Dayside convection and auroral morphology during an interval of northward interplanetary magnetic field, Ann. Geophys., 18, 436.

Newell, P. T., C.-I. Meng, D. G. Sibeck, and R. Lepping (1989), Some lowaltitude cusp dependencies on the interplanetary magnetic field, J. Geophys. Res., 94, 8921.

Onsager, T. G., and S. A. Fuselier (1994), The location of magnetic reconnection for northward and southward interplanetary magnetic field, in Solar System Plasmas in Space and Time, Geophys. Monogr. Ser., vol. 84, edited by J. L. Burch and J. H. Waite Jr., p. 183, AGU, Washington, D. C.

Onsager, T. G., C. A. Kletzing, J. B. Austin, and H. MacKiernan (1993), Model of magnetosheath plasma in the magnetosphere: Cusp and mantle particles at low altitudes, Geophys. Res. Lett., 20, 479. 
Reiff, P. H., and J. L. Burch (1985), IMF By-dependent plasma flow and Birkeland currents in the dayside magnetosphere: 2. A global model for northward and southward IMF, J. Geophys. Res., 90, 1595.

Sandel, B. R., and A. L. Broadfoot (1981), Morphology of Saturn's aurora, Nature, 292, 679.

Sandel, B. R., et al. (1982), Extreme ultraviolet observations from the Voyager 2 encounter with Saturn, Science, 215, 548

Sandholt, P. E., C. J. Farrugia, S. W. H. Cowley, M. Lester, and J.-C. Cerisier (2001), Excitation of transient lobe cell convection and auroral arc at the cusp poleward boundary during a transition of the interplanetary magnetic field from south to north, Ann. Geophys., 19, 487.

Trauger, J. T., et al. (1998), Saturn's hydrogen aurora: Wide field and planetary camera 2 imaging from the Hubble Space Telescope, J. Geophys. Res., 103, 20,237.
Vasyliunas, V. M. (1983), Plasma distribution and flow, in Physics of the Jovian Magnetosphere, edited by A. J. Dessler, p. 395, Cambridge Univ. Press, New York

S. V. Badman, E. J. Bunce, and S. W. H. Cowley, Department of Physics and Astronomy, University of Leicester, University Road, Leicester LE1 7RH, UK

J. T. Clarke, Center for Space Physics, Boston University, 725 Commonwealth Avenue, Boston, MA 02215, USA.

J.-C. Gérard and D. Grodent, Laboratoire de Physique Atmosphérique et Planétaire, Université de Liège, Allee du 6 Aout, Liège B-4000, Belgium. (jc.gerard@ulg.ac.be; d.grodent@ulg.ac.be) 

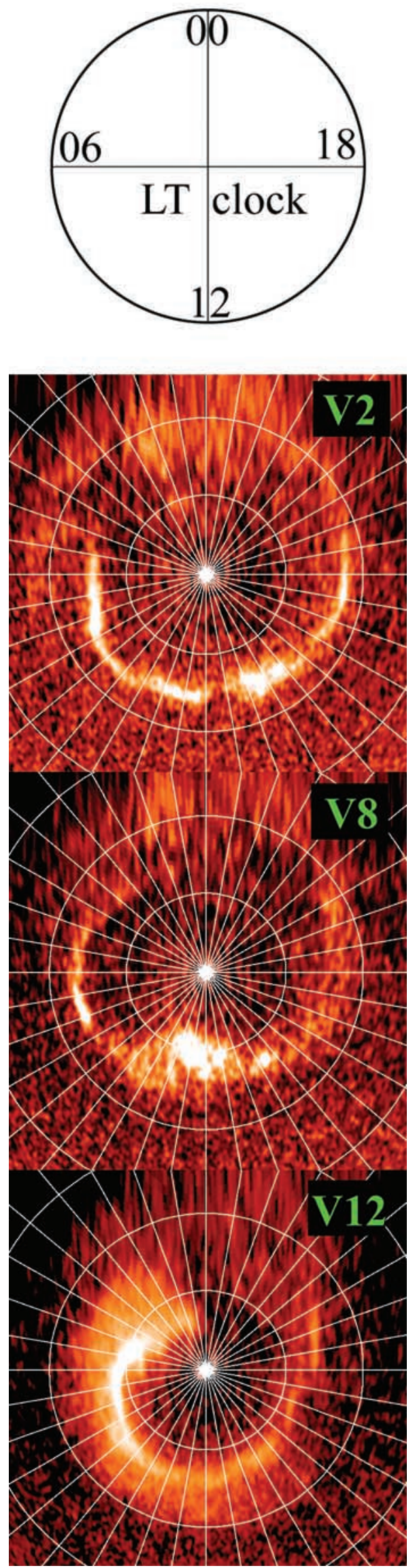

Figure 3. Polar (orthographic) projections of HST-STIS images of Saturn's south FUV aurora. Images were taken on (top) 10 January (V2), (middle) 21 January (V8), and (bottom) 28 January (V12) 2004 and belong to the low-field rarefaction period, the recovery period following the 15 January solar wind compression, and the recovery period following the 25 January interplanetary shock, respectively. The image has been projected on a polar view where the south is seen by a virtual observer above the north pole, looking through the planet. In these viewing conditions, the south pole is at the center, the direction of the Sun (1200 LT) is conveniently oriented towards the bottom, dawn (0600 LT) to the left, dusk (1800 LT) to the right as indicated in the LT clock panel. The parallels are separated by $10^{\circ}$ of latitude and the meridians by $10^{\circ}$ of longitude. 


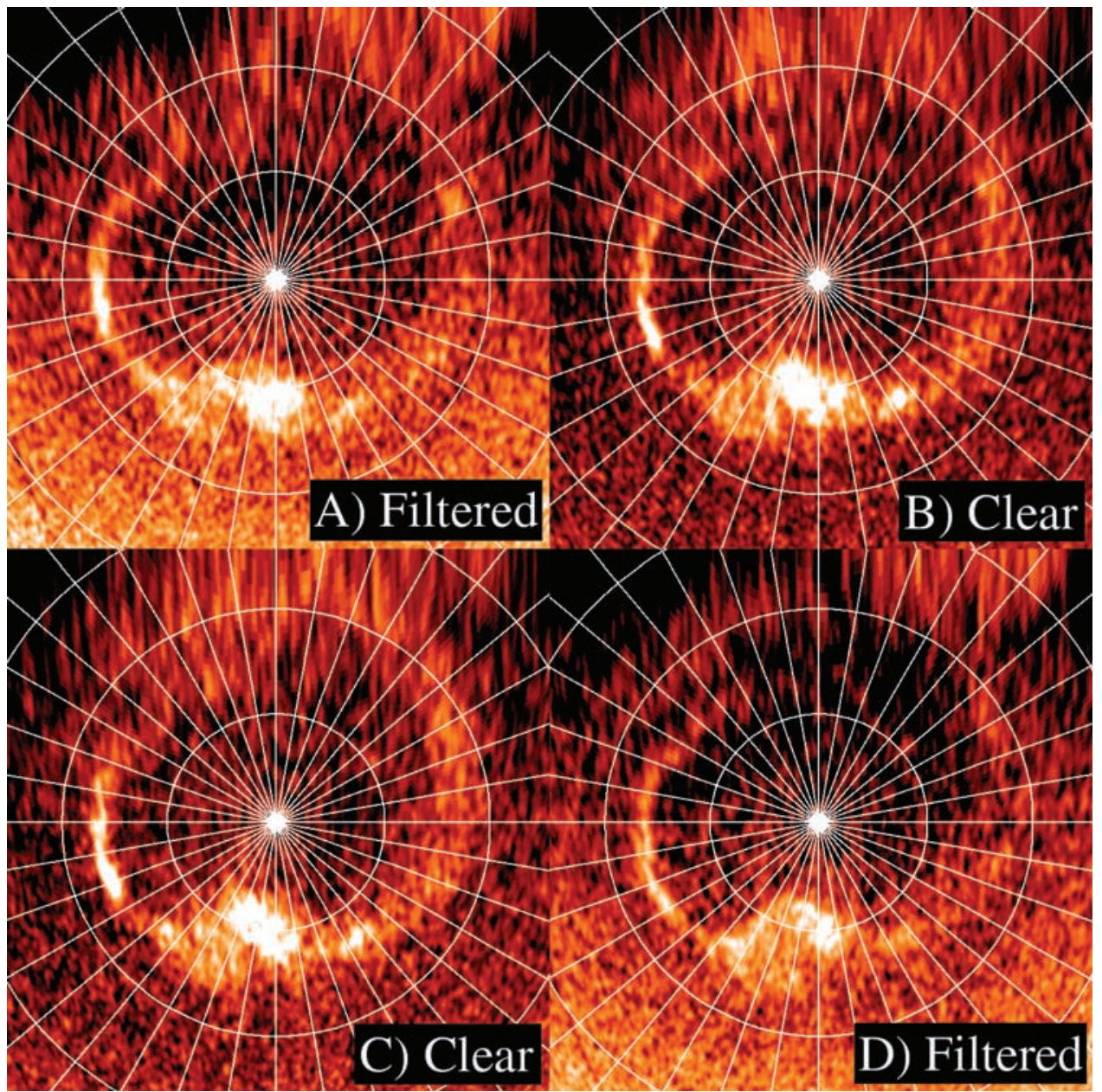

Figure 4. Polar projections of HST-STIS images of Saturn's southern hemisphere UV aurora taken on 21 January 2004 (Visit 8). (a) and (d) Images were taken in the SrF2 'filtered' mode; (b) and (c) images were unfiltered. 


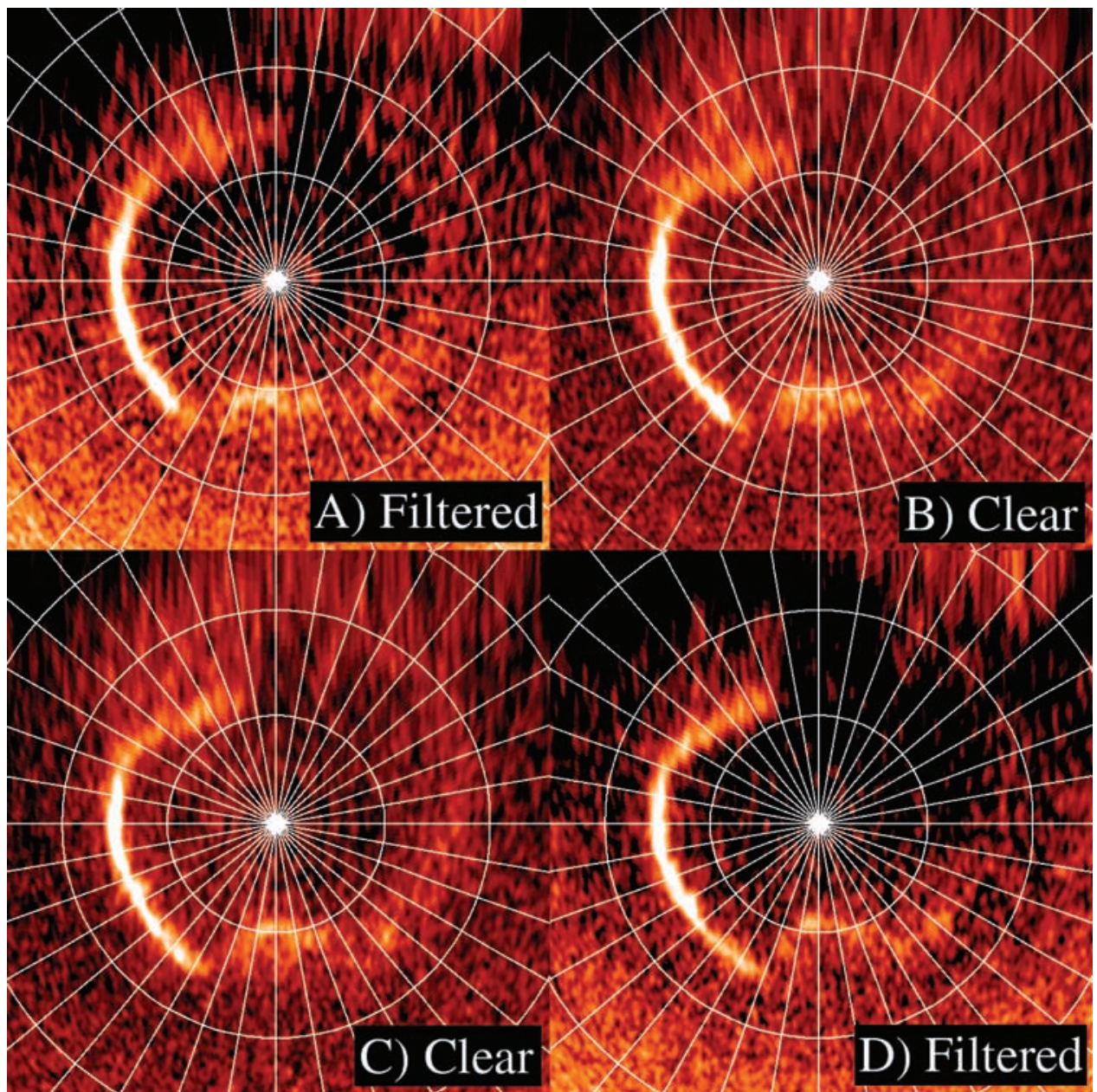

Figure 5. Polar projections of HST-STIS images of Saturn's southern hemisphere UV aurora taken on 20 January 2004 (Visit 7). 


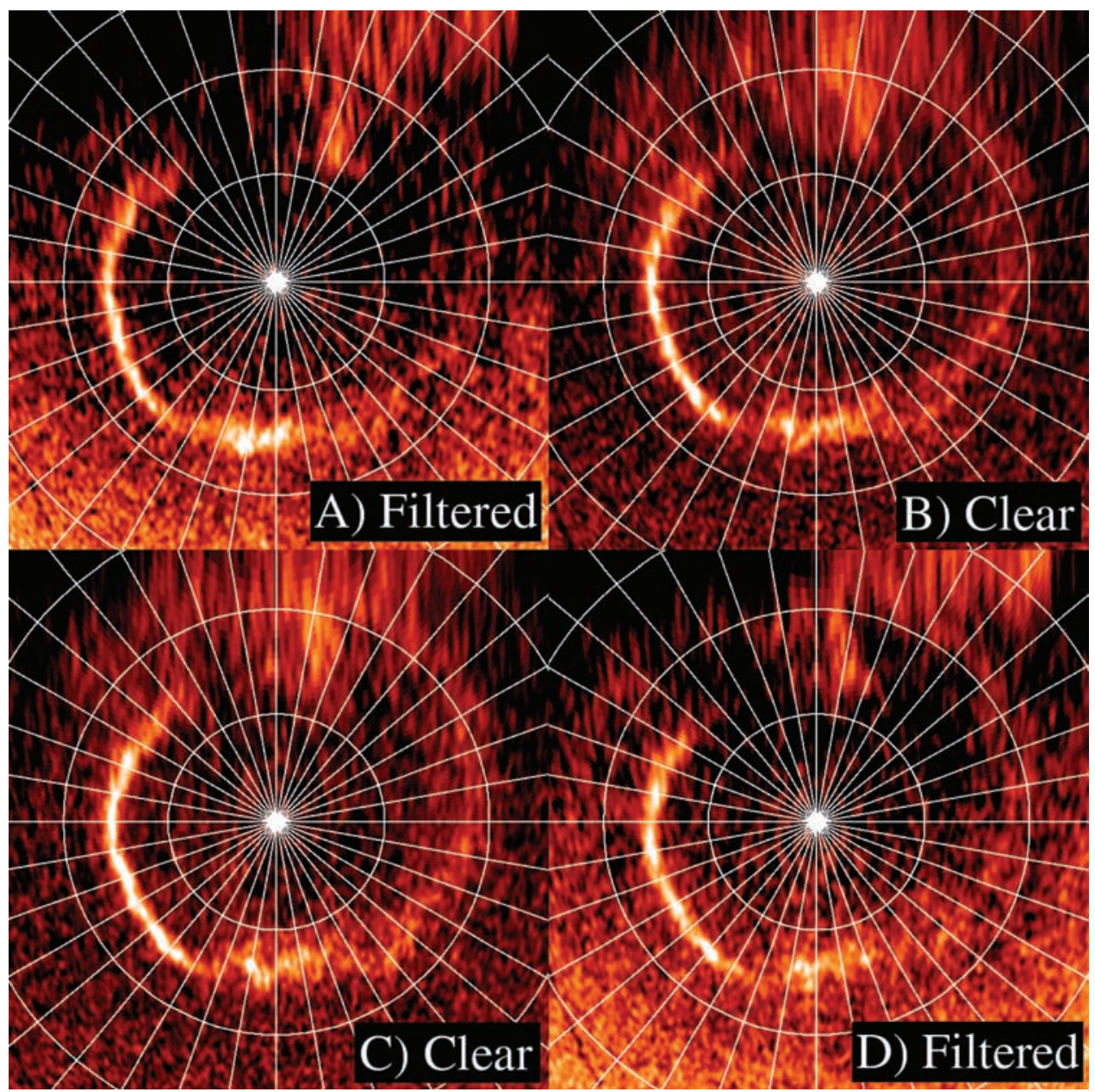

Figure 6. Polar projections of HST-STIS images of Saturn's southern hemisphere UV aurora taken on 23 January 2004 (Visit 9). 